\title{
An enhancement of path selection to cluster head based on multi-hop routing in two-tier wireless sensor network
}

\author{
Wan Isni Sofiah Wan Din ${ }^{1}$, Asyran Zarizi Bin Abdullah ${ }^{2}$, Razulaimi Razali ${ }^{3}$, Ahmad Firdaus $^{4}$, \\ Salwana Mohamad ${ }^{5}$, Danakorn Nincarean Eh Phon ${ }^{6}$, Cik Feresa Mohd Foozy ${ }^{7}$ \\ 1,2,4,5,6Faculty of Computer Systems and Software Engineering, Universiti Malaysia Pahang, Malaysia \\ ${ }^{3}$ Faculty of Computer and Mathematical Sciences, Universiti Teknologi MARA, Malaysia \\ ${ }^{7}$ Faculty of Computer Science and Information Technology, Universiti Tun Husssein Onn, Malaysia
}

\begin{abstract}
Article Info
Article history:

Received Jan 27, 2019

Revised Apr 18, 2019

Accepted Jun 11, 2019

\section{Keywords:}

Cluster head

Multi-hop routing

Path selection

Two tier

Wireless sensor network

ABSTRACT

Wireless Sensor Network (WSN) is a distributed wireless connection that consists many wireless sensor devices. It is used to get information from the surrounding activities or the environment and send the details to the user for future work. Due to its advantages, WSN has been widely used to help people to collect, monitor and analyse data. However, the biggest limitation of WSN is about the network lifetime. Usually WSN has a small energy capacity for operation, and after the energy was used up below the threshold value, it will then be declared as a dead node. When this happens, the sensor node cannot receive and send the data until the energy is renewed. To reduce WSN energy consumption, the process of selecting a path to the destination is very important. Currently, the data transmission from sensor nodes to the cluster head uses a single hop which consumes more energy; thus, in this paper the enhancement of previous algorithm, which is MAP, the data transmission will use several paths to reach the cluster head. The best path uses a small amount of energy and will take a short time for packet delivery. The element of Shortest Path First (SPF) Algorithm that is used in a routing protocol will be implemented. It will determine the path based on a cost, in which the decision will be made depending on the lowest cost between several connected paths. By using the MATLAB simulation tool, the performance of SPF algorithm and conventional method will be evaluated. The expected result of SPF implementation will increase the energy consumption in order to prolong the network lifetime for WSN.
\end{abstract}

Copyright () 2019 Institute of Advanced Engineering and Science. All rights reserved.

\section{Corresponding Author:}

Wan Isni Sofiah Wan Din,

Faculty of Computer Systems and Software Engineering,

Universiti Malaysia Pahang,

26300 Gambang, Pahang, Malaysia.

Email: sofiah@ump.edu.my

\section{INTRODUCTION}

To date, the implementations of Wireless Sensor Network (WSN) have become more challenging and exciting. Many research were conducted to prolong the lifetime of the sensor network. WSN contains many distributed devices or nodes that are connected to the gateway by using a sensor for collecting, tracking, and monitoring a wide range of applications [1,2]. Previously, the conventional wired technology was used, but there were some limitations. It only operates with a fixed connection and limited coverage distance. The cost for installation and maintenance were also expensive as compared to the wireless communication.

The WSN implementation is expected to overcome the wired issues. The network is more scalable and flexible to realise the important potential roles of Internet of Things (IoTs) [3, 4]. It runs a lot of 
applications to help the user to get the real time information and system control, such as getting information of floods in flooded area, checking the air quality in kindergarten, sensoring high temperatures and automatically activate the air conditioner and track the nearest petrol station while driving. Remarkably, all these applications could be operated by WSN at a low cost and wide distance coverage [5].

The group of IEEE 802 protocol will be used as the standard that allows wireless communication [6]. Previously, single hop transmission were used to send and receive the node to and from the cluster head before forwarding the packet to the sink/base station, which is usually connected to the Internet [7]. A base station receives the packet, and collects the data for future computation. Implementations of multi hop transmission with network clustering technique were used to increase the performance and optimise the WSN lifetime [8]. In WSN the connection is made between the sensor node to the cluster head, and from cluster head to the base station. However, there are several constraints that need to be considered for implementing WSN, which is the energy usage, and the time taken to send and receive the packet [9]. Both issues will affect the node lifetime. In the single hop routing transmission, energy consumption is the main issue. The nodes that are closer to the cluster head have a longer lifetime as compared to the node that is located far from the cluster head. This is because the node needs to carry a packet in heavy traffic for a long distance [10]. It will waste node energy and increase the time for sending and receiving the packet. The battery will drain fast and once the energy is less than the threshold value the sensor node will be considered as a dead node. Therefore, it is no more available for data collection until the battery is changed. The continuity of this situation will affect the whole process because if more dead nodes are found in WSN, then a high delay in packet delivery will happen [11].

Subsequently, a solution is needed to meet the requirement of the fast packet delivery and low delay transmission to save more energy and render a longer lifetime of sensor nodes. Hence, an improvement of path selection by using multi-hop routing technique will solve the issues. It will find the best path to send and receive the packet to the cluster head. The selection is based on a lower cost of the path in network clustering. It will make more effective on energy usage and short time in packet delivery in order to increase the WSN lifetime. In designing a WSN, the throughput and energy consumption is extremely important [12]. According to [13], WSN executes around 800 to 1000 instructions, and if they fail to do so, then they will affect the node lifetimes, and thus unable to provide an efficient performance for packet delivery. In WSN cases, the bottlenecks issues are related to the limited supply of energy because most of the sensor nodes are equipped with non-charging batteries. When the sensor node is out of battery, then it will be considered as dead. The charging energy of the sensor node is a challenge and sometimes it is impossible to do because of the difficulty to reach the WSN area of placement. Due to these issues, the development of WSN is putting priority on energy saving for designing the WSN software and hardware components.

a. Existing Routing Protocols

Ad Hoc On-Demand Distance Vector (AODV) is a protocol of unicast and multicast routing that is used to transmit the packet between the source and destination. AODV contains a Router Request Broadcast (RREQ), Router Reply Propagation (RREP) and Router Error Message (RERR) as a message used for distributing and selecting a path for packet transmission [7]. Figure 1 explains about the transmission between source (S) and destination (D). The RREQ is a process of finding a path from source to destination. In this situation, the destination is the cluster head. The source node will distribute a message request to all connected nodes in the WSN and if the node is not a destination, then the message will be forwarded to another node until it finds the destination. The RREP will forward a message from the destination node to the source node after RREQ is successfully received by a destination. It is used to inform the source node and make an establishment of the connection. The connection will continue until the source node runs out of energy, then RERR will be used to inform all nodes that the connection is not available and the other source nodes need to flood the message of RREQ to find a new path to the cluster head.
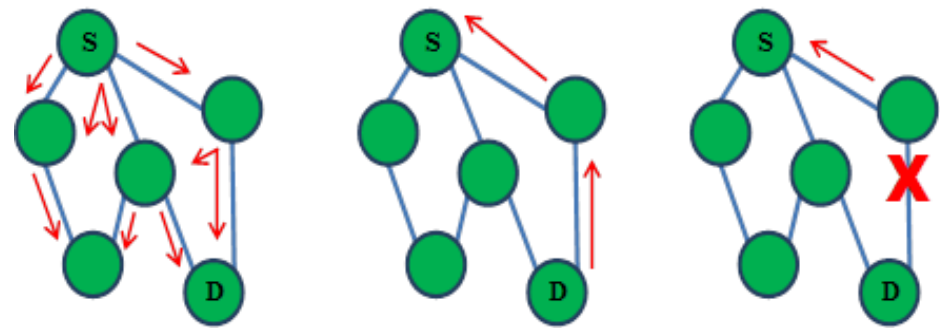

Figure 1. AODV protocol message 
The Dynamic Source Routing (DSR) is an on-demand routing protocol in a reactive topology-based protocol. It is built for a wireless multi-hop ad hoc mobile network and applies two types of routing mechanisms, which is route discovery and route maintenance [14]. For DSR route mechanisms, the source Node A must connect to a destination Node D, then it will send a request to the neighbouring node. Then, Node B will receive the request, but if Node B path has been stored in a path record before, it will discard the new request. Node B will forward the request message to next hop node. The same goes to Node $\mathrm{C}$ i.e. until destination Node $\mathrm{D}$ receives the request. When node $\mathrm{D}$ receives the request message from source Node A then it will reply back to the router which contains a path between the sources Node A to destination Node D. But if the request message failed during transmission, the node will send a Router Error to the source node, the source will refer to the last resort of receiver node and forward the request message again. Figure 2 shows the process on how DSR works.

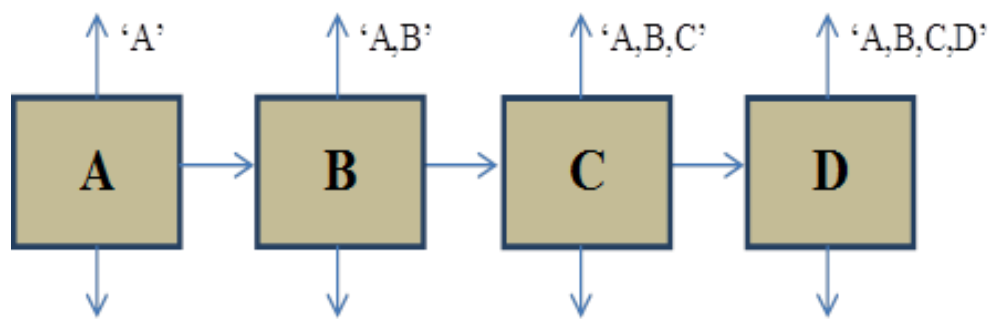

Figure 2. DSR Route Mechanisms

The SPF Algorithm is used in Open Shortest Path First (OSPF) to find the best path from a source to a destination [15]. The route will discover all the possible paths to reach a destination, then the information of metric for each path will be stored in a router database, it will be arranged from the shortest to the longest path. Figure 3 shows how SPF Algorithm works, and it will calculate the cost from source to destination, then each of the calculation result will be stored in the SPF Table.

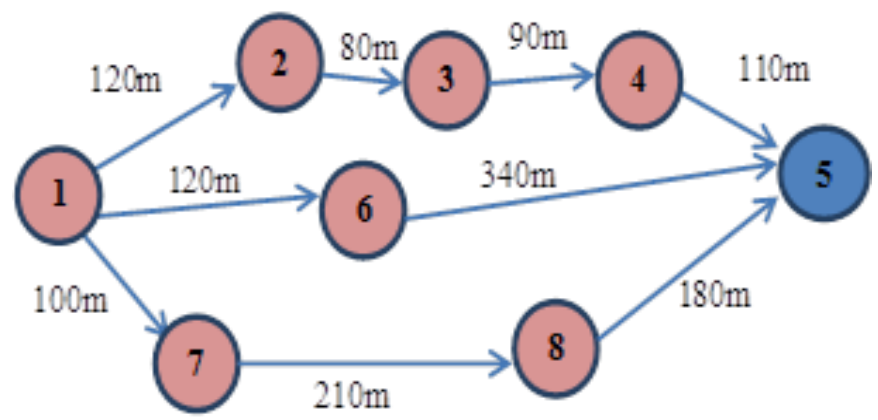

Figure 3. SPF Path Calculation

The best path (which has a lowest metric) will be located in the first row as a main path. However, if the main path connection has a problem (logical or physical problem), then SPF algorithm will automatically use a second path in the table as the new route until the main path is repaired. This process is used to avoid any disruptions during packet transmission.

b. MAP Protocols

Figure 4 shows the MAP algorithm for data transmission from sensor node to the cluster head. In MAP, the data transmitted from sensor node to the cluster head will use a single hop or direct transmission. The sensor node did not choose the best path to reach the cluster head. As shown in Figure 4, each node and algorithm will check if the node is still alive, then the nodes can send the data directly to the cluster head. This differs with enhancement of the proposed shortest path first, where the nodes will choose the best path and shortest path to reach their cluster head. 


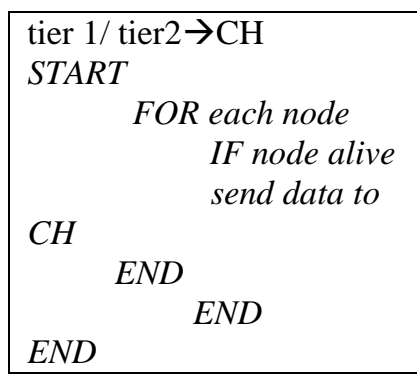

Figure 4. MAP data transmission algorithm

The rest of this paper is organized as follows. Section 2 explains research method which is the enhancement from direct transmission to the multi-hop transmission. Section 3 discussed about the hypothesis for the enhancement approach and lastly, Section 4 concludes the overall enhancement of MAP in transmitting data from the sensor node to the cluster head.

\section{RESEARCH METHOD}

Figure 5 shows the overview of MAP implementation [16]. In MAP, the sensor node uses a single hop to communicate with their respective cluster head. Based on this proposed approach, an enhancement will be made for the sensor node to communicate with the cluster head. Multi-hop communication based on SPF was chosen to send the data to the cluster head. In MAP algorithm, the node will be randomly distributed in sensor field $100 \times 100$ metre by using basic formula area of circle $\left(A=\pi \times r^{2}\right)$ [17] . The algorithm will track the location (coordinates) of each distributed node to determine the distance between node and cluster head for the path selection to the destination. MAP uses fuzzy logic to select a cluster head. The parameters that are involved in MAP are remaining energy, cost and centralisation of the node. Then, WSN will measure the node lifetime to see the maximum energy consumption used by each sensor node in the network. The measurement will look at the first and last dead node. Based on this measurement information, the SPF algorithm will be used to improve the node lifetime and a comparison should be done to see how long energy could be saved by using this new approach.

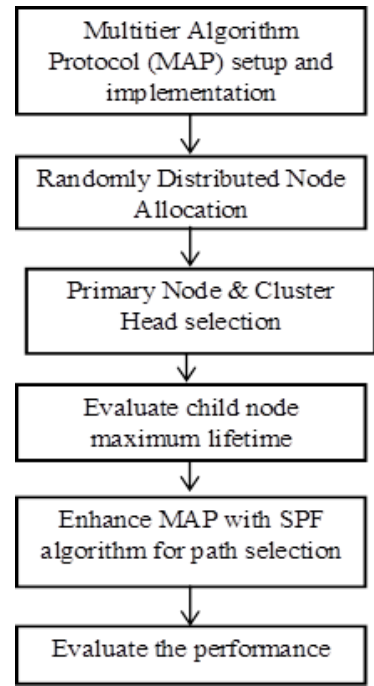

Figure 5. Enhancement of MAP implementation

Figure 6 is the design and implementation of the existing MAP. In MAP, it contains a base station, cluster head, sensor nodes and primary nodes. As in Figure 5, the sensor nodes transmit the data directly to the cluster head but for the proposed approach, the sensor nodes will transmit the data by using a multi-hop communication. This is to identify and analyse the energy consumption of the sensor node while transmitting the data; hence, prolongs the network lifetime. 


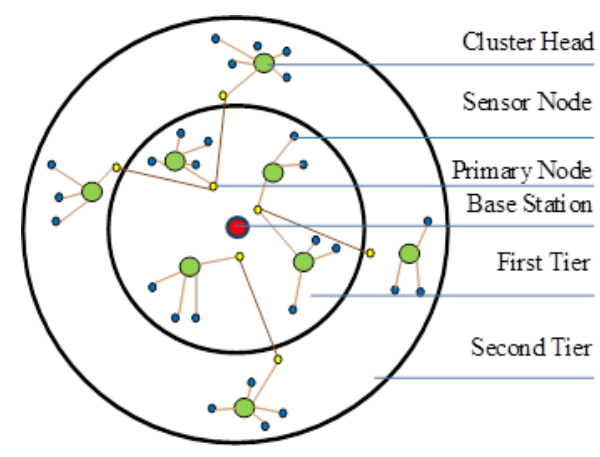

Figure 6. MAP design and implementation

The focus of this paper is on multi-hop routing technique for path selection in order to reduce energy usages and make a fast delivery to extend the sensor node lifetime. To achieve these goals, it is important to highlight the study area and its boundaries, which are explained as follows.

The Multi-tier Protocol (MAP) [17] algorithm will be used to operate multi-hop routing in WSN, and the parameters as shown in Table 1 involved are 100 static nodes that will be randomly located in a 2-tier network clustering. The node will be set up with the same energy level with the multiple load size traffic (10 Mbps, $30 \mathrm{Mbps}, 50 \mathrm{Mbps}$ ) and multiple operation time (10 minutes, 20 minutes, 30 minutes) to measure time taken for a node to transmit the packet and the maximum node lifetime which can be recorded. Since the research focuses on the sensor node, the existing cluster head selection by using Fuzzy Logic will be used [17]. The implementation will use MATLAB as a simulation tool. The route calculation uses SPF algorithm to find the best path for sending and receiving data to reduce energy usage, and fast delivery message to make long lifetimes for WSN.

Table 1. MAP parameters [16]

\begin{tabular}{ll}
\hline Parameter & Value \\
\hline Network Coverage & $100 \times 100 \mathrm{M}$ \\
Base Station Location & 50,50 \\
Number of nodes & 100 \\
Energy $(\mathrm{J})$ & $1,0 / 5<\mathrm{E}<2$ \\
Iteration & 9999 \\
\hline
\end{tabular}

The improvement of path selection should go through a comparison between the current implementation and the new approach of path selection. It is important to prove that the use of a new approach can produce better results than ever before. These comparisons need to use the same parameters to measure the energy consumption for maximum single node lifetime and the last surviving node in the network.

Figure 7 shows a flow process in selecting a path by using SPF algorithm. In this paper, the SPF algorithm is used as a medium to select a path from a source to the destination. At the beginning, SPF will discover a possible path that is connected to the source. Then, it will choose a path which has the lowest cost. SPF will do some comparisons between the possible paths, to identify the path with the lowest cost. If the path $\mathrm{X}$ has the lowest cost as compared to other paths, then it will be selected as the main path, however if path X's cost is higher than the main path, then it will become a backup path, for the main path. If the main path has a problem that makes the connection disconnected, then the backup path will take over the transmission as the main path. All path directions to the destination will be stored in the SPF memory. The conversional methods will randomly select the path and it will waste everything.

Figure 8 shows how the SPF calculates the cost based on distance value. Node A will be declared as a source node. The calculation will start at Node A to all nodes connected to A. The source Node A has neighbours which is Node $\mathrm{B}$ and Node $\mathrm{C}$. The distance taken from source to $\mathrm{A}$ is 0 and will add the distance value of Node $B$ direction which is $0+9=9$, then the other path from $A$ to $C$ is $0+8=8$. Now both path must be compared to determine the lowest cost between $\mathrm{A}$ to $\mathrm{B}$, and $\mathrm{A}$ to $\mathrm{C}$. If $\mathrm{A}$ to $\mathrm{C}$ is of lowest value, then the data will be stored at the WSN memory as a main path and the process will continue until it reaches to the destination node as shown in Figure 8 and Table 2. The selection of a best path for all nodes is shown in Table 2. For example, the shortest path from Node A to I is 30 , so to know the path, it need to look 
backwards. Starting from Node I and the shortest path that came from Node H, the shortest path from $\mathrm{H}$ to A was through Node G, and the shortest path from Node G to A was through Node E, then Node E to A was over the Node D and from D to A was via C. The shortest path from Node C to Node A was the direct path which is $\mathrm{C}$ to $\mathrm{A}$. So the path will be $\mathrm{A} \rightarrow \mathrm{C} \rightarrow \mathrm{D} \rightarrow \mathrm{E} \rightarrow \mathrm{G} \rightarrow \mathrm{F} \rightarrow \mathrm{H} \rightarrow \mathrm{I}$. The value of the path cost 30; however by using a conversional method, the path may increase the cost. For example, the path from Node A to Node I is using the random path selection, $A \rightarrow B \rightarrow E \rightarrow G \rightarrow H \rightarrow I$. Which is from A to B are (9), from $B$ to $E$ (6), from E to $\mathrm{G}$ (7) from $\mathrm{G}$ to $\mathrm{H}$ (11), and from $\mathrm{H}$ to I (5). The total cost will become 38, which is higher than by using SPF algorithm. The higher cost is recorded from path selection, and then the longest time needed to deliver the message, will also increase the energy usage and make a short node lifetime. This will adversely affect the overall network performance of WSN.

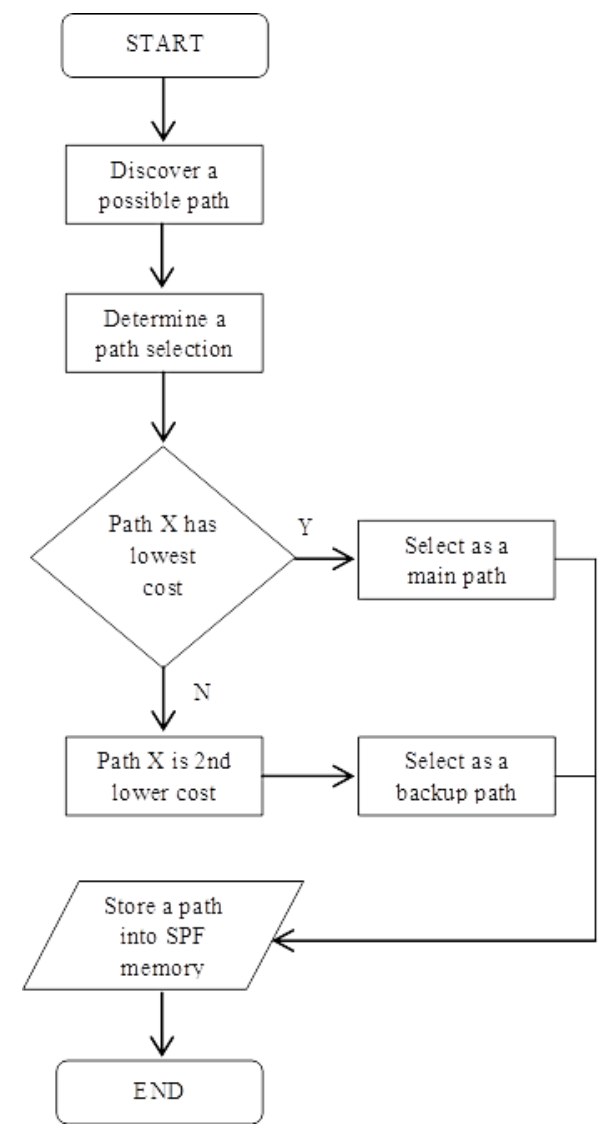

Figure 7. Flow chart for SPF

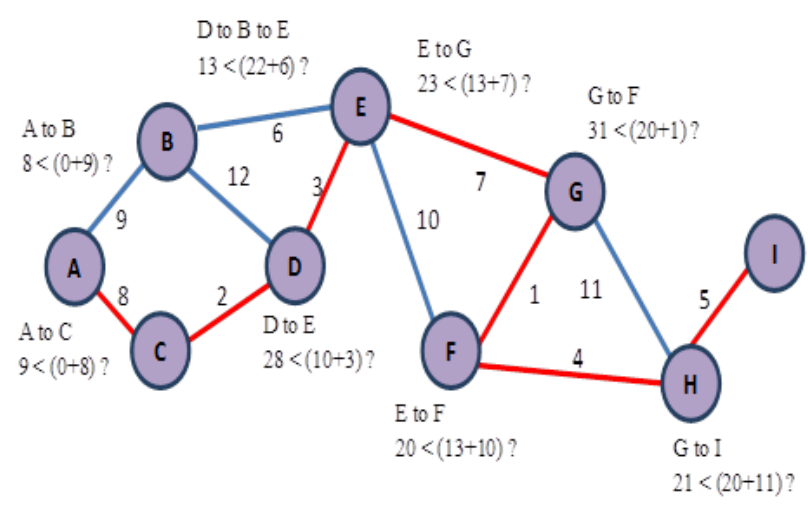

Figure 8. SPF calculation 


\begin{tabular}{ccc} 
& Table 2.The SPF value \\
\hline Node & Cost & Connected Node \\
\hline$\square$ & 0 & - \\
$B$ & 9 & $\mathrm{~A}$ \\
$C$ & 8 & $\mathrm{~A}$ \\
$D$ & 10 & $\mathrm{C}$ \\
$\mathrm{E}$ & 13 & $\mathrm{D}$ \\
$\mathrm{F}$ & 21 & $\mathrm{G}$ \\
$G$ & 20 & $\mathrm{E}$ \\
$H$ & 25 & $\mathrm{~F}$ \\
$I$ & 30 & $\mathrm{H}$ \\
\hline
\end{tabular}

\section{RESULTS AND DISCUSSION}

For performance evaluation, the proposed enhancement, which is SPF, will be compared with the MAP algorithm in terms of energy consumption while transmitting the data. In this hypothesis, performance evaluation will involve two techniques, as shown in Figure 9. Technique A will use the existing algorithm; MAP without changing any parameters and the energy consumption for the first running will be collected. Technique B is the proposed technique where SPF will be used to transmit data to the cluster head by using a multi-path as compared to Technique A which only used direct transmission to the cluster head. Both techniques will use 100 and 200 sensor nodes with 2,000, 4,000, 6,000, 8,000 and 10,000 bits of data. This is to measure the differences and the consistency of the proposed enhancement and the energy usage while transmitting data.

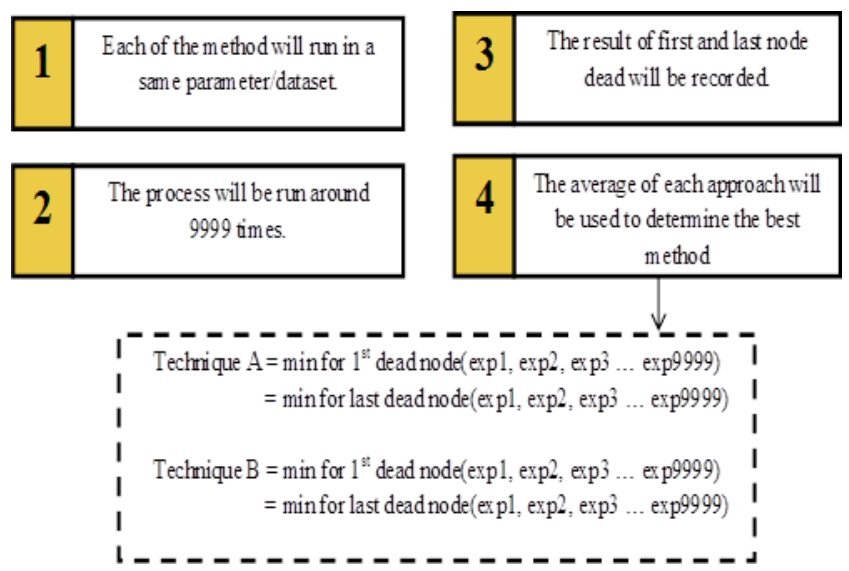

Figure 9. Evaluation performance for two approaches

The current MAP algorithm will add some function of SPF in order to enable the algorithm of selecting a multi-hop path. The main path selection has the lowest cost to provide an efficient energy usage. The performance of the current implementation and enhancement algorithm will be compared to look at how much it can be improved and fulfill the requirement as needed.

Initially, both methods will run around 9,999 times by using the same dataset, as mentioned in Table 1. The results for the first and the last dead node will be captured. Based on the existing MAP algorithm and the proposed enhancement of data transmission, the results later will identify the improvement of the energy usage for multi-hop transmission instead of single hops for a 2-tier network design.

\section{CONCLUSION}

Every day, the implementation of using WSN is rapidly increasing, and it will keep increasing in the next few years because of flexibilities and cost efficiencies [18]. The WSN is easily made for users to perform their job, such as collecting data, tracing a location and analysing information. However, there are some issues that will reduce the WSN performance which is energy usage, and the time consumed to send and receive the packet [19]. Generally, the WSN placements are difficult to access, when the WSN energy runs out, the nodes are unable to send and receive the information from other nodes. The enhancement of path selection by using a multi-hop transmission will reduce the WSN energy consumption. The SPF 
algorithm is proposed to overcome the highlighted issues, in which the algorithm will find the shortest cost in their connected paths to the destination. Later, the proposed enhancement in data transmission from direct to multi-path will be run in MATLAB. By proposing this, it will reduce the transmission time for sending and receiving; hence, prolonging the WSN lifetime. The effectiveness of this approach will be measured by comparing the energy consumption while transmitting the data and it must show that the proposed approach can contributed towards effective energy consumption.

\section{ACKNOWLEDGEMENTS}

The author would like to thank UMP RDU Grants (RDU-180364) in providing the financial support and facilities for this study. We are also grateful and would like to acknowledge and thank the Faculty of Computer Systems \& Software Engineering UMP and Systems Network \& Security Research Group (Sysnets) that has supported this research.

\section{REFERENCES}

[1] L. Muduli, et al., "Wireless Sensor Network Based Fire Monitoring In Underground Coal Mines: A Fuzzy Logic Approach," Process Safety and Environmenta Protection, vol. 113, pp. 435-447, 2018.

[2] M. Elhoseny, et al., "Optimizing K-Coverage Of Mobile WSNs," Journal of Industrial Information Integration, vol. 92, pp. 142-153, 2018.

[3] Y. H. Lin, et al., "Optimal And Maximized Configurable Power Saving Protocols For Corona-Based Wireless Sensor Networks," IEEE Transaction Mobile Computing, vol. 14, pp. 2544-2559, 2015.

[4] M. Burhanuddin, et al., "A Review on Security Challenges and Features in Wireless Sensor Networks: IoT Perspective," Journal Telecommunication Electronic and Computer Engineering, vol. 10, pp. 17-21, 2018.

[5] I. Del Castillo, et al., "Hardware Platform for Wide-Area Vehicular Sensor Networks With Mobile Nodes," Vehicular Communication, vol. 3, pp. 21-30, 2016.

[6] S. G. Santhi and B. Divya, "Energy Consumption Using Ieee802.15.4 Sensor Networks," International. Journal of Computer Applications, vol. 116, pp. 30-33, 2015.

[7] T. C. W. Mohammed A. Mahdi, "Performance Evaluation Of Single-Path And Multipath Manets Routing Protocols For Dense And Sparse Topology," International Journal Software Engineering and Computer System, vol. 3, pp. 31-42, 2017.

[8] P. Kuila and P. K. Jana, "Energy Efficient Load-Balanced Clustering Algorithm for Wireless Sensor Networks," Procedia Technology, vol. 6, pp. 771-777, 2012.

[9] B. A. Bakr and L. T. Lilien, "Extending Lifetime of Wireless Sensor Networks by Management of Spare Nodes," Procedia Computer Sciences, vol. 34, pp. 493-498, 2014.

[10] P. Sharma and A. Gupta, "A Review on Multiple Single Hop Clustering Based Data Transmission in Wireless Sensor Network," International Journal of Engineering and Computer Science, vol. 3, 2014

[11] A. A. J. Al-Sabbagh, et al., "Effects Of Mobility Models And Nodes," Int. J. Ad Hoc, Sens. Ubiquitous Comput., vol. 5, pp. 8, 2014.

[12] C. Tunca, et al., "Ring Routing: An Energy-Efficient Routing Protocol for Wireless Sensor Networks With A Mobile Sink," IEEE Transition and Mobile Computing, vol. 14, pp. 1947-1960, 2015.

[13] S. Branch and R. Branch, "Recognition And Correction Of Flexible Error Using Multi-Level Redundant Residue Number," Journal of Theoritical and Application Information Technology, vol. 64, pp. 484-489, 2014.

[14] U. Nagaraj and P. P. Dhamal, "Performance Evaluation of Proactive and Reactive Protocols in Vanet," International Journal Infrastructure Education and Technology, vol. 2, pp. 434-438, 2012

[15] F. L. Lugayizi, et al., "Performance Evaluation Of Ospf And Eigrp Routing Protocols For Video Streaming Over Next Generation Networks," International Journal of Advanced Computer Sciences, vol. 4, pp. 29-35, 2015.

[16] W. I. S. W. Din, et al., "Developing Multi-Tier Network Design for Effective Energy Consumption of Cluster Head Selection in WSN," Scientific Research Journal, vol. 13, pp. 1-11, 2016.

[17] W. I. S. W. Din, et al., "Map: The New Clustering Algorithm Based On Multitier Network Topology To Prolong The Lifetime Of Wireless Sensor Network," IEEE 10th International Colloquium Signal Process and Its Applications, CSPA, pp. 173-177, 2014.

[18] A. A. Allahham and M. A. Rahman, "A Smart Monitoring System For Campus Using Zigbee Wireless Sensor Networks," International Journal of Software Engineering and Computer System, vol. 4, pp. 1-14, 2018

[19] H. Kumar and P. K. Singh, "Node Energy Based Approach to Improve Network Lifetime and Throughput in Wireless Sensor Networks," Journal of Telecommunication Electronic and Computer Engineering Architecture, vol. 9, pp. 83-88, 2018. 